\title{
Predicting Acute Kidney Injury Following Non-Emergent Cardiac Surgery: A Preoperative Scorecard
}

\author{
Ahmed Mokhtar ${ }^{1}$, Karthik Tennankore ${ }^{1}$, Steve Doucette $^{2}$, and Christine Herman ${ }^{3}$ \\ ${ }^{1}$ Dalhousie University Faculty of Medicine \\ ${ }^{2}$ Nova Scotia Health Authority \\ ${ }^{3}$ Dalhousie University
}

October 14, 2020

\begin{abstract}
Objective: To determine the predictors of postoperative AKI following non-emergent cardiac surgery among patients with variable preoperative eGFR levels. Methods: Retrospective study of patients who underwent elective or in-hospital cardiac surgical procedures performed between January 2006 and November 2015. The procedures included isolated CABG, isolated AVR or combined CABG and AVR. The primary outcome AKI (any stage) following non-emergent cardiac surgery utilizing the 2012 KDIGO criteria. Patients were categorized based the following renal outcomes: mild AKI, severe AKI (KDIGO stage 2 or 3) and post-operative dialysis.. Results: A total of 6713 patients were included in our study. The mean age was 66.8 years ( $\mathrm{SD} \pm 10.3$ ), with $76.2 \%$ being males. A total of 4487 patients had normal or mildly decreased eGFR (G1 or G2) preoperatively $(66.8 \%)$, while 1960 patients were in the G3 category (29.1\%). Only 266 patients (3.9\%) had G4 or worse renal function. A total of 1489 (28.5\%) patients experienced post-operative AKI. The need for postoperative dialysis occurred in $4.2 \%$ of the AKI subgroup. In-hospital mortality was higher among the AKI subgroup ( $7.3 \%$ vs $0.5 \%, \mathrm{p}<0.0001)$. In an adjusted model, a lower pre-operative eGFR category was the strongest predictor of AKI. A practical scorecard for the preoperative estimation of severe AKI for non-emergent cardiac procedures incorporating these parameters was developed. Conclusions: Preoperative eGFR is the strongest predictor of post-operative AKI in individuals undergoing non-emergent cardiac surgery. A practical scorecard incorporating preoperative predictors of AKI may allow informed decision making and to predict AKI following non-emergent cardiac surgery
\end{abstract}

\section{Introduction:}

Perioperative acute kidney injury (AKI) is a major determinant of both short-term and long-term outcomes following cardiac surgery ${ }^{1-4}$. AKI has been has been shown to be an independent predictor of morbidity and mortality in the post-operative period, including adverse cardiovascular events and need for dialysis ${ }^{5-7}$. Depending on the definition, post-operative AKI can occur in up to $30 \%$ of patients undergoing cardiac surgery ${ }^{8,9}$. Approximately $5 \%$ of patients with AKI following cardiac surgery will require dialysis ${ }^{3}$, a subgroup that suffers from dismal outcomes with mortality rates approaching $60 \%$ in some studies and a high burden on health care resources ${ }^{10-13}$.

While the majority of patients undergo cardiac surgery electively, a significant proportion of patients undergo in-hospital surgery during index admission due to a variety of causes, including acute coronary syndrome (ACS) or exacerbation of heart failure, which can increase the risk of AKI ${ }^{14,15}$. Furthermore, while there is extensive literature in assessing the risk of AKI following cardiac surgery, only a few studies have aimed to address the impact of pre-operative CKD (utilizing established criteria) on post-operative AKI and predicting the need for dialysis following cardiac surgery ${ }^{16-18}$. In addition, rarely has the issue been examined solely in a lower-risk, non-emergent population, which may represent a significantly different population with distinctive outcomes $^{19}$. 
Therefore, the objective of this current study is to identify the predictors of post-operative acute kidney injury and specifically, to determine if CKD stage is associated with an incremental increase in the risk of AKI among a cohort of Canadian, elective and non-urgent in-hospital patients undergoing cardiac surgery.

\section{Material and Methods:}

\section{Patient Population:}

The study population included patients who underwent elective or in-hospital cardiac surgical procedures performed between January 2006 and November 2015 at the Queen Elizabeth II Health Sciences Center (QEII HSC) in Halifax, Nova Scotia, Canada. The QEII HSC is the only cardiac surgical center for the Province of Nova Scotia serving a base population of 971,395. We included all patients undergoing the following cardiac surgical procedures at the QE-II-HSC: isolated coronary artery bypass grafting (CABG) surgery, isolated aortic valve replacement (AVR), or combined CABG and AVR. Patients were excluded if they underwent an emergency or urgent (defined as the need for operative intervention within 24hrs) surgery, endocarditis, mitral valve procedures and complex procedures, such thoracic aortic surgery and trauma. Cardiac surgery was performed with cardiopulmonary bypass and anticoagulation was achieved using intravenous heparin given at a dose of $400 \mathrm{IU} / \mathrm{kg}$ with a target activated clotting time (ACT) greater than 450 seconds. Antifibrinolytic agents were used routinely and consisted mainly of tranexamic acid. Intermittent cold blood cardioplegia was delivered in an antegrade or retrograde fashion based on surgeon preference. Protamine sulfate was given for reversal of heparin in all patients.

\section{Data Source:}

Data was obtained using the using the Maritime Heart Center (MHC) Cardiac Surgery Registry. The MHC registry is a detailed prospectively collected clinical database containing perioperative data on all cardiac surgery cases at the Queen Elizabeth Health Sciences Centre (QEII HSC) and commenced in $1995^{20}$.

Primary Outcome, determination of Preoperative eGFR and post-operative AKI:

The primary outcome was the development of AKI (any stage) following cardiac surgery utilizing the 2012 KDIGO criteria (Table 1$)^{21}$. Severe AKI was defined as patients fulfilling stage 2 or stage 3 AKI, while postoperative dialysis was defined as patients requiring dialysis regardless of stage. Additional outcomes of clinical significance included mortality and the need for post-operative dialysis. Preoperative eGFR was calculated based on the Kidney Disease-Improving Outcomes 2012 Guidelines ${ }^{22}$ (Table 2). The following formula was used to calculate eGFR as per the CKD-EPI equation: GFR $=141 * \min (\operatorname{Scr} / x, 1) \alpha * \max (\mathrm{Scr} / x, 1)-1.209 *$ 0.993 Age * 1.018 [if female]. For analytical purposes, G1 and G2 patients were assessed together while all other eGFR categories were analyzed separately.

\section{Variable Selection and analysis:}

Other preoperative clinical characteristics of interest included age, sex, chronic obstructive pulmonary disease (COPD), peripheral and/or cerebral vascular disease, New York Heart Association (NYHA) functional class, diabetes, redo surgery, clamp time, cardioplegia type and completeness of revascularization. Variable selection was based on multiple models predicting postoperative AKI in cardiac surgery that have been both internally and externally validated ${ }^{23}$. Individual procedure types were categorized as follows: isolated CABG, isolated AVR and combined AVR/CABG.

\section{Statistical Analysis}

Continuous variables were compared using a 2-tailed t test or Wilcoxon rank sum test, and categorical variables were analyzed using a chi-square or Fisher's exact test, as appropriate.

Adjusted logistic regression models were generated to examine the association of AKI with pre-operative eGFR and pre-operative surgical status. Variables to be included in the model included sex, age, diabetes, chronic obstructive pulmonary disease, COPD, left ventricular ejection fraction (LVEF), Peripheral vascular disease, cerebrovascular disease, glomerular filtration rate (eGFR), type of surgery and previous cardiac 
surgery. A bedside post-operative scorecard for predicting AKI was then generated then utilizing the results of the logistic regression. This was developed by determining the potential strength of individual relationships between the previously identified variables based on the method described by Sullivan et al ${ }^{24}$. Scores for each factor were assigned relative weights of each coefficient in the multivariable regression model. Initial scores were assigned an integer value based on the $B$-coefficient of the OR, and the scores were subsequently adjusted up or down by increments of 0.5 to ensure that the sum of the risk scores corresponded to the category of predicted risk.

All statistical analyses were performed using SAS software version 9.4 (The SAS Institute, Cary, NC).

Ethics

This study was conducted with the full approval of the institutional (Nova Scotia Health Authority) Research Ethics Board.

\section{Results:}

\section{Study population}

A total of 6713 patients were included in our study. Table 3 demonstrates the baseline characteristics of the cohort. The mean age was 66.8 years $(\mathrm{SD} \pm 10.3)$ and men accounted for the majority of the study population (76.2\%). A total of 4487 patients had normal or mildly decreased eGFR (G1 or G2) preoperatively (66.8\%), while 1960 patients were in the G3 category (29.1\%). Only 266 patients (3.9\%) had G4 or worse renal function. A total of $2852(42.5 \%)$ of patients had NYHA class III or IV. The spectrum of surgical interventions performed included the following: isolated CABG $(\mathrm{n}=4766,71 \%)$, isolated AVR $(\mathrm{n}=1251$, 18.6\%), combined AVR and CABG ( $\mathrm{n}=696,10.4 \%)$. Among these, $3192(47.5 \%)$ were elective, and 3521 $(52.2 \%)$ were determined to be urgent. A total of 1489 (28.5\%) patients experienced post-operative AKI. Patients who developed postoperative AKI were more likely to have reduced preoperative eGFR. Those with G3 or higher CKD were at a significantly higher risk of post-operative AKI. In addition, patients with AKI tended to be older (mean age $69.6, \pm 10$ ), and had higher rates of comorbidities, including diabetes (50\% vs $35.2 \%, \mathrm{p}<0.0001)$ and COPD (19.3\% vs $11.6 \%, \mathrm{p}<0.0001)$. In contrast, the risk of AKI was no different comparing elective versus in-house cardiac surgery. The AKI subgroup had a higher rate of combined procedures $(16.7 \%$ vs $8.6 \%, \mathrm{p}<0.0001)$.

\section{Postoperative Outcomes:}

Unadjusted postoperative outcomes are shown in Table 4. The majority of patients with AKI were classified as stage 1 AKI $(94.6 \%)$. The need for postoperative dialysis occurred in $4.2 \%$ of the AKI subgroup. Inhospital mortality was higher among the AKI subgroup ( $7.3 \%$ vs $0.5 \%, \mathrm{p}<0.0001)$. The incidence of low cardiac output syndrome reintubation, tamponade, prolonged ventilation was also markedly higher among the AKI subgroup.

\section{Independent Predictors of Renal Outcomes:}

Predictors of AKI, severe AKI and post-operative AKI, along with odds ratios (OR) and confidence limits (CI) are shown in Figure 1. In an adjusted model, a lower pre-operative eGFR category was the strongest predictor of AKI. For the G3 category, there was a significant association with any AKI (OR 2.11, 95\% CI $1.84-2.41$ ), but not with severe AKI or post-operative dialysis. In contrast, the G4 category predicted any AKI (OR 4.29, 95\% CI 3.22 - 5.71), severe AKI (OR 23.97, 95\% CI 11.5 - 49.95) and post-operative dialysis (OR 14.83, 95\% CI $6.8-32.33$ ). G5 CKD had the strongest association with all renal outcomes (any AKI; OR 69.65, 95\% CI 16.61 - 292.08, severe AKI; OR 385.4, 95\% CI 150.5 - 986.66 and postoperative dialysis; OR $124.1,95 \%$ CI $49.4-311.73)$.

Other independent predictors of the need for post-operative dialysis included COPD (OR 2.24, 95\% CI 1.2 - 4.12), diabetes (OR 2.45, 95\% CI 1.38 - 4.54) and LVEF <30\% (OR 2.64, 95\% CI 0.86 - 3.08). In contrast, age, male sex, redo sternotomy and complexity of procedure were not predictive of severe AKI or DIALYSIS in our cohort. 


\section{Scorecard for Preoperative Estimation of Severe AKI Risk for Non-emergent Cardiac Procedures}

A practical scorecard for the preoperative estimation of severe AKI for non-emergent cardiac procedures is noted in Table 5. As shown in Table 5b, using these scores, risk estimates for severe AKI ranged from $1 \%$ (for scores of 0-3.5) to $>90 \%$ (for scores [?]13.5).

\section{Discussion:}

In this study, we analyzed post-operative AKI among a large cohort of patients undergoing non-emergent cardiac surgery. As expected, patients with AKI were more likely to have predisposing risk factors that have been reported in the literature including advanced age, CKD, diabetes, chronic lung disease and peripheral vascular disease. Following adjustment for clinical differences, we found that a lower pre-operative eGFR was the strongest predictor of any AKI, severe AKI and need for post-operative dialysis among patients undergoing elective and in-hospital cardiac surgery. While age, female sex, redo sternotomy and complexity of procedure were not independently predictive of severe AKI or need for dialysis in our cohort (in contrast to other models $2,12,23,25-28$ ), Importantly, we were able to combine these predictors (along with low eGFR) into a pragmatic risk prediction tool that may have practical implications for the care of patients requiring non-emergent cardiac surgery.

Our study expands on the existing literature around risk prediction for AKI following cardiac surgery. Predicting major adverse outcomes post-operatively is a vital aspect of patient-centered care with shared decision making. This is even more crucial given the demographics of patients who require cardiac surgery; the increase in the proportion of frail and multi-comorbid patients that require cardiac surgery further emphasizes the need for better risk prediction. ${ }^{29}$. Unlike prior studies, we opted to focus solely on a low-risk, non-emergent population. Acknowledging that emergency surgery is an established risk factor for AKI and that decisions around emergency surgery may not provide the same time frame for patient discussion, our risk prediction model is more focussed on clinical scenarios where the discussion of risks may have a greater impact on patient choice. ${ }^{30}$.

Outside of the selection of a lower risk cohort, the results of our study need to be compared to other literature looking at risk prediction for AKI following cardiac surgery. Historically post-operative AKI has been defined by utilizing absolute serum creatinine, a $50 \%$ or greater increase in serum creatinine from baseline or need for dialysis (AKI-D) ${ }^{7}$. Various criteria to define postoperative AKI have been utilized ${ }^{31,32}$. However, a consensus has been established to utilize the KDIGO criteria in order to achieve more consistent and reproducible results when assessing postoperative AKI. Among stable, elective patients, eGFR represents a more accurate reflection of kidney function and is less susceptible to other confounders, such as sex, age and weight ${ }^{33}$. The prevalence of patients with moderate renal dysfunction (G3 and worse) among our cohort was 33.1\%, which approximates the larger Society of Thoracic Surgery (STS) database (prevalence of 27.1\%) accounting for the differences in the urgency of surgery and the use of the MDRD formula to calculate eGFR 3 . The inclusion of CKD in our model is significant, as it has been established as most important predictor in prior models that included preoperative $\mathrm{GFR}^{19,27,33}$. As expected in our cohort, preoperative CKD was the highest predictor of poor renal outcomes, with category G4 being threshold for independent prediction of severe AKI and the need for dialysis following cardiac surgery.

With regards to postoperative outcomes, the incidence of post-operative AKI within our population followed the expected published frequency (5-28\%), accounting for variability in definitions of $\mathrm{AKI}^{8,9}$. However, the rate of postoperative dialysis among our study cohort was lower as compared to the rates reported in other centers ${ }^{19,34-36}$. Multiple explanations underpin this finding, including the exclusion of more complicated procedures and the lower overall clamp time among the non-AKI group.

Our scorecard for predicting severe AKI is has several clinical implications. To our knowledge, it was derived from the largest known cohort of exclusively elective cardiac surgery procedures, improving generalizability. Furthermore, the scorecard integrates exclusively pre-operative variables, emphasizing its value as a bedside tool to identify patients at risk for AKI in advance of surgery to allow the provision of perioperative clinical strategies to mitigate the risk of renal injury. 
To our knowledge, only a few existing studies have utilized scorecards in predicting AKI among non-emergent cardiac surgery patients. Callejas et al enrolled 942 electively undergoing cardiac surgery among a multicentre Spanish cohort ${ }^{26}$. This scorecard was externally validated against the Thakar model, despite the inclusion of emergency cases. Interestingly, preoperative creatinine determination and eGFR displayed no statistical significance in predicting post-operative AKI. Palomba et al showed that in a prospective cohort of 603 patients undergoing elective cardiac surgery, the incidence of AKI was $11 \%(n=66)$, with $18 \%$ of those patients requiring dialysis $(n=11) \cdot{ }^{37}$. However, the inclusion of intraoperative and postoperative variables in this study precludes the use of their derived scoring tool (the Acute Kidney Injury following Cardiac Surgery score) prior to determining the need for surgery.

Our study has several strengths. In addition to being a large cohort of non-emergent cardiac patients, this is one of few studies that have formally utilized the KDIGO criteria (an established AKI stratification tool) following elective cardiac surgery to predict postoperative $\mathrm{AKI}^{26,38}$. In addition, our derived scorecard is truly "bedside" where all the required variables to provide a risk-estimate are pre-operative.

There are limitations to this study that warrant further discussion. Due to the retrospective design, there is an inherent risk of bias and residual confounding. We acknowledge that a points-based risk-scoring derived from $B$-coefficient models may be limited due to competing risks, namely, mortality ${ }^{39}$. Furthermore, our risk prediction model and scorecard require external validation prior to widespread use. We relied solely on serum creatinine to classify AKI as per the KDIGO criteria, (rather than urine output), and this may have led to an underestimate of the outcome of AKI. However, urine output is poorly documented in the reported literature of post-operative patients ${ }^{40}$. In addition, we acknowledge that race was not used in the calculation of eGFR, due to a lack of data. However, given the known demographics of the Nova Scotia population, this is unlikely to have led to a large error in the observed association. Although race was not able to be included in the eGFR calculation (as it is not routinely captured in the MHC), only a paucity of individuals in Nova Scotia are African descendants based on 2016 census data, (21,910 individuals (2.4\%) out of 908,340 Nova Scotians) ${ }^{41}$. Nonetheless, the results of this study may not be generalizable to populations with a larger proportion of African descendants. Finally, this study was derived from a single centre, rather than a multicenter study, which may limit external validity.

\section{Conclusion:}

In this study we identified that preoperative eGFR remains the biggest predictor of all stages of postoperative AKI in individuals undergoing non-emergent cardiac surgery. A practical scorecard incorporating preoperative predictors of AKI and the need for dialysis may allow informed decision making and provision of strategies to prevent AKI following non-emergent cardiac surgery.

\section{References:}

1. Kangasniemi O-P, Mahar MAA, Rasinaho E, et al. Impact of estimated glomerular filtration rate on the 15-year outcome after coronary artery bypass surgery. Eur J Cardiothorac Surg . 2008;33(2):198-202. doi:10.1016/j.ejcts.2007.11.002

2. Mehta RH, Grab JD, O'Brien SM, et al. Bedside tool for predicting the risk of postoperative dialysis in patients undergoing cardiac surgery.Circulation . 2006;114(21):2208-2216. doi:10.1161/CIRCULATIONAHA.106.635573

3. Cooper WA, O'Brien SM, Thourani VH, et al. Impact of renal dysfunction on outcomes of coronary artery bypass surgery: Results from the Society of Thoracic Surgeons National Adult Cardiac Database.Circulation . 2006;113(8):1063-1070. doi:10.1161/CIRCULATIONAHA.105.580084

4. Hillis GS, Croal BL, Buchan KG, et al. Renal function and outcome from coronary artery bypass grafting: Impact on mortality after a 2.3-year follow-up. Circulation . 2006;113(8):1056-1062. doi:10.1161/CIRCULATIONAHA.105.591990

5. Howell NJ, Keogh BE, Bonser RS, et al. Mild renal dysfunction predicts in-hospital mortality 
and post-discharge survival following cardiac surgery. Eur J Cardio-thoracic Surg . 2008;34(2):390-395. doi:10.1016/j.ejcts.2008.04.017

6. Elmistekawy E, McDonald B, Hudson C, et al. Clinical Impact of Mild Acute Kidney Injury After Cardiac Surgery. Ann Thorac Surg . 2014;98(3):815-822. doi:10.1016/j.athoracsur.2014.05.008

7. Zakeri R, Freemantle N, Barnett V, et al. Relation between mild renal dysfunction and outcomes after coronary artery bypass grafting.Circulation . 2005;112(9 SUPPL.). doi:10.1161/CIRCULATIONAHA.104.522623

8. Mariscalco G, Lorusso R, Dominici C, Renzulli A, Sala A. Acute kidney injury: A relevant complication after cardiac surgery. Ann Thorac Surg . 2011;92(4):1539-1547. doi:10.1016/j.athoracsur.2011.04.123

9. Rosner MH, Okusa MD. Acute kidney injury associated with cardiac surgery. Clin J Am Soc Nephrol . 2006;1(1):19-32. doi:10.2215/CJN.00240605

10. Chertow GM, Levy EM, Hammermeister KE, Grover F, Daley J. Independent association between acute renal failure and mortality following cardiac surgery. Am J Med . 1998;104(4):343-348. doi:S0002934398000588 [pii]

11. Landoni G, Zangrillo A, Franco A, et al. Long-term outcome of patients who require renal replacement therapy after cardiac surgery.Eur J Anaesthesiol . 2006;23(1):17-22. doi:10.1017/S0265021505001705

12. Huen SC, Parikh CR. Predicting acute kidney injury after cardiac surgery: A systematic review. Ann Thorac Surg . 2012;93(1):337-347. doi:10.1016/j.athoracsur.2011.09.010

13. Dasta JF, Kane-Gill SL, Durtschi AJ, Pathak DS, Kellum JA. Costs and outcomes of acute kidney injury (AKI) following cardiac surgery.Nephrol Dial Transplant . 2008;23(6):1970-1974. doi:10.1093/ndt/gfm908

14. Parikh S V, de Lemos JA, Jessen ME, et al. Timing of In-Hospital Coronary Artery Bypass Graft Surgery for Non-ST-Segment Elevation Myocardial Infarction Patients. JACC Cardiovasc Interv . 2010;3(4):419427. doi:10.1016/j.jcin.2010.01.012

15. McNeely C, Markwell S, Vassileva C. Trends in Patient Characteristics and Outcomes of Coronary Artery Bypass Grafting in the 2000 to 2012 Medicare Population. Ann Thorac Surg . 2016;102(1):132-138. doi:10.1016/J.ATHORACSUR.2016.01.016

16. Birnie K, Verheyden V, Pagano D, et al. Predictive models for kidney disease: Improving global outcomes (KDIGO) defined acute kidney injury in UK cardiac surgery. Crit Care . 2014;18(6):1-20. doi:10.1186/s13054-014-0606-x

17. Kristovic D, Horvatic I, Husedzinovic I, et al. Cardiac surgery-associated acute kidney injury: Risk factors analysis and comparison of prediction models. Interact Cardiovasc Thorac Surg . 2015;21(3):366373. doi:10.1093/icvts/ivv162

18. Machado MN, Nakazone MA, Maia LN. Lesão renal aguda baseada nos critérios KDIGO (Kidney Disease: Improving global outcomes) em pacientes com creatinina sérica elevada submetidos à cirurgia cardíaca.Brazilian J Cardiovasc Surg . 2014;29(3):299-307. doi:10.5935/1678-9741.20140049

19. Pannu N, Graham M, Klarenbach S, et al. A new model to predict acute kidney injury requiring renal replacement therapy after cardiac surgery. Cmaj . 2016;188(15):1076-1083. doi:10.1503/cmaj.151447

20. Herman CR, Buth KJ, Kent B a, et al. Clopidogrel increases blood transfusion and hemorrhagic complications in patients undergoing cardiac surgery. Ann Thorac Surg . 2010;89(2):397-402. doi:10.1016/j.athoracsur.2009.10.051

21. Kellum J a, Lameire N, Aspelin P, et al. KDIGO Clinical Practice Guideline for Acute Kidney Injury. Kidney Int Suppl . 2012;2(1):1-138. doi:10.1038/kisup.2012.7

22. ***. Kdigo. Kidney Int Suppl . 2013;3(1):4-4. doi:10.1038/kisup.2012.76 
23. Huen Sarah PCR. Predicting Acute Kidney Injury Following Cardiac Surgery: A Systematic Review. Ann Thorac Surg . 2013;93(1):337-347. doi:10.1016/j.athoracsur.2011.09.010.Predicting

24. Sullivan LM, Massaro JM, D'Agostino RBS. Presentation of multivariate data for clinical use: The Framingham Study risk score functions. Stat Med . 2004;23(10):1631-1660. doi:10.1002/sim.1742

25. Ortega-Loubon C, Fernández-Molina M, Carrascal-Hinojal Y, Fulquet-Carreras E. Cardiac surgeryassociated acute kidney injury.Ann Card Anaesth . 2016;19(4):687-698. doi:10.4103/0971-9784.191578

26. Callejas R, Panadero A, Vives M, et al. Preoperative predictive model for acute kidney injury after elective cardiac surgery: A prospective multicenter cohort study. Minerva Anestesiol . 2019;85(1):34-44. doi:10.23736/S0375-9393.18.12257-7

27. Demirjian S, Schold JD, Navia J, et al. Predictive models for acute kidney injury following cardiac surgery. Am J Kidney Dis . 2012;59(3):382-389. doi:10.1053/j.ajkd.2011.10.046

28. Thakar C V., Arrigain S, Worley S, Yared JP, Paganini EP. A clinical score to predict acute renal failure after cardiac surgery. J Am Soc Nephrol . 2005;16(1):162-168. doi:10.1681/ASN.2004040331

29. Gainer RA, Curran J, Buth KJ, David JG, Légaré JF, Hirsch GM. Toward Optimal Decision Making among Vulnerable Patients Referred for Cardiac Surgery: A Qualitative Analysis of Patient and Provider Perspectives. Med Decis Mak . 2017;37(5):600-610. doi:10.1177/0272989X16675338

30. Wang Y, Bellomo R. Cardiac surgery-associated acute kidney injury: Risk factors, pathophysiology and treatment. Nat Rev Nephrol . 2017;13(11):697-711. doi:10.1038/nrneph.2017.119

31. Mehta RL, Kellum JA, Shah S V, et al. Acute Kidney Injury Network: report of an initiative to improve outcomes in acute kidney injury. Crit Care . 2007;11(2):R31. doi:10.1186/cc5713

32. Bellomo R, Ronco C, Kellum JA, Mehta RL, Palevsky P. Acute renal failure - definition, outcome measures, animal models, fluid therapy and information technology needs: the Second International Consensus Conference of the Acute Dialysis Quality Initiative (ADQI) Group.Crit Care . 2004;8(4):R204-12. doi: $10.1186 / \mathrm{cc} 2872$

33. Nadim MK, Forni LG, Bihorac A, et al. Cardiac and vascular surgery-associated acute kidney injury: The 20th International Consensus Conference of the ADQI (Acute Disease Quality Initiative) Group. $J$ Am Heart Assoc . 2018;7(11). doi:10.1161/JAHA.118.008834

34. Sutherland L, Hittesdorf E, Yoh N, Lai T, Mechling A, Wagener G. Acute kidney injury after cardiac surgery: A comparison of different definitions. Nephrology . 2019;(July):1-7. doi:10.1111/nep.13669

35. Howitt SH, Grant SW, Caiado C, et al. The KDIGO acute kidney injury guidelines for cardiac surgery patients in critical care: A validation study. BMC Nephrol . 2018;19(1):1-8. doi:10.1186/s12882-018-0946-x

36. Machado MN, Nakazone MA, Maia LN. Prognostic value of acute kidney injury after cardiac surgery according to kidney disease: Improving global outcomes definition and staging (KDIGO) criteria. PLoS One . 2014;9(5):1-7. doi:10.1371/journal.pone.0098028

37. Palomba H, De Castro I, Neto ALC, Lage S, Yu L. Acute kidney injury prediction following elective cardiac surgery: AKICS Score. Kidney Int . 2007;72(5):624-631. doi:10.1038/sj.ki.5002419

38. Crosina J, Lerner J, Ho J, et al. Improving the Prediction of Cardiac Surgery-Associated Acute Kidney Injury. Kidney Int Reports . 2017;2(2):172-179. doi:10.1016/j.ekir.2016.10.003

39. Austin PC, Lee DS, D'Agostino RB, Fine JP. Developing points-based risk-scoring systems in the presence of competing risks. Stat Med . 2016;35(22):4056-4072. doi:10.1002/sim.6994

40. McIlroy DR, Argenziano M, Farkas D, Umann T, Sladen RN. Incorporating oliguria into the diagnostic criteria for acute kidney injury after on-pump cardiac surgery: Impact on incidence and outcomes. $J$ Cardiothorac Vasc Anesth . 2013;27(6):1145-1152. doi:10.1053/j.jvca.2012.12.017 
41. Statistics-Canada. Diversity of the Black population in Canada: An overview. 2019:1-22.
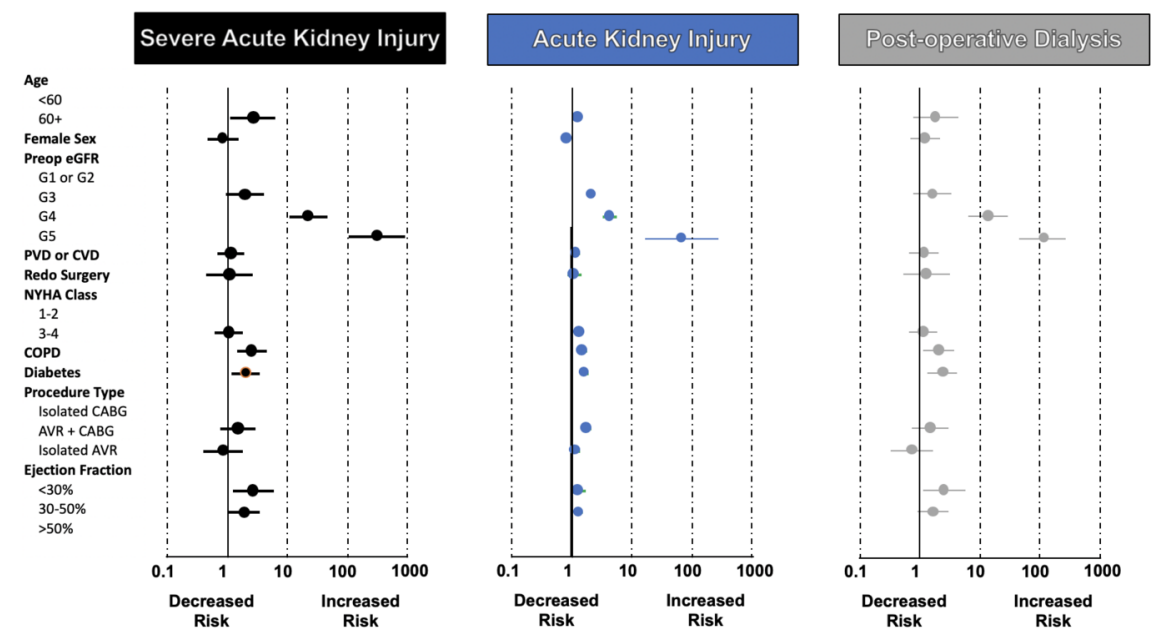

Figure 1. Adjusted predictors of AKI, severe AKI and need for post-operative dialysis after multivariable adjustment (visualized as relative odds with $95 \%$ confidence intervals).

Table 1. Staging of AKI as per Kidney Disease-Improving Outcomes (KDIGO) 2012 Guidelines

AKI Stage

Stage 1

Stage 2

Stage 3
Table 1. Staging of AKI Definition

Increase in serum creatin Increase in serum creatin Increase in serum creatin

Table 2. Staging of GFR as per Kidney Disease-Improving Outcomes 2012 Guidelines utilizing the CKD-E]

\section{GFR category}

G1

G2

G3a

G3b

G4

G5

Table 3. Baseline characteristics of patients categorized based on AKI following non-emergent cardiac surgery.

Variable

Age (yrs), Means \pm SD

$<60$

60-69

70-79

80

Female

KDIGO staging (PREoperative eGFR) using CKD-EPI formula instead of RIFLE

G1 or G2
Table 3. B Overall ( $66.8 \pm 10$. $1610(24 \%$ $2297(34.2$ $2077(30.9$ $729(10.9 \%$ $1600(23.8$

$4487(66.8$ 
Table 3. Baseline characteristics of patients categorized based on AKI following non-emergent cardiac surgery.

G3

G4

G5

PVD \&/or CVD, N (\%)

Redo N, (\%)

Intra op IABP N (\%)

NYHA

Class I or II

Class III or IV

COPD

Diabetes

Active endocarditis

Procedure Type

Isolated CABG

Isolated AVR

AVR + CABG

Cardioplegia Type

No Cardioplegia

Antegrade Only

Retrograde Only

Both

Urgency

Elective

In-house

Clamp Time

$>90 \mathrm{~min}$

Incomplete Revascularization
Table 3. B

$1960(29.2$

$228(3.4 \%)$

$38(0.6 \%)$

$1539(22.9$

$369(5.5 \%)$

$57(0.8 \%)$

3861 (57.5

$2852(42.5$

$893(13.3 \%$

$2586(38.5$

$19(0.3 \%)$

$4766(71 \%$

$1251(18.6$

$696(10.4 \%$

$68(1 \%)$

$4811(71.8$

$466(7 \%)$

$1351(20.2$

$3192(47.5$

$3521(52.5$

$2144(31.9$

$53(0.8 \%)$

CABG - Coronary artery bypass grafting, CKD- Chronic Kidney Disease Epidemiology Collaboration, COPD - chronic obstructive lung disease, CVD - cerebrovascular disease, IABP - intra-aortic balloon pump, NYHA - New York Heart association functional class, PVD peripheral vascular disease

Table 4. Unadjusted postoperative outcomes among AKI vs non-AKI subgroups

\author{
AKI Stage \\ 0 \\ 1 \\ 2 \\ 3 \\ Postoperative dialysis \\ In-hospital Mortality \\ Low Cardiac Output Syndrome \\ Reoperation for Bleeding \\ Postoperative Tamponade \\ Reintubation \\ Prolonged Ventilation \\ Transfusion of Blood Products
}

Table 4. Unadjusted postoperative outcc

AKI $(\mathbf{n = 1 4 8 9})$
$0(0 \%)$
$1409(94.6 \%)$
$7(0.5 \%)$
$73(4.9 \%)$
$63(4.2 \%)$
$108(7.3 \%)$
$272(18.3 \%)$
$122(8.2 \%)$
$86(5.8 \%)$
$130(8.7 \%)$
$508(34.1 \%)$
$818(54.9 \%)$


Table 5. Scorecard for Preoperative Estimation of Severe AKI Risk After Non-emergent Cardiac Surgery 5a. Predictors of Severe AKI

Risk Factor

Age (y)

$60+$

GFR

G3

G4

G5

PVD CVD

COPD

Diabetes

AVR + CABG

$\mathrm{EF}$

$<30 \%$

$30-50 \%$
Table 5. Scorece 5a. Predictors o Score

1

9

0.5

1.5

1

0.5

1.5
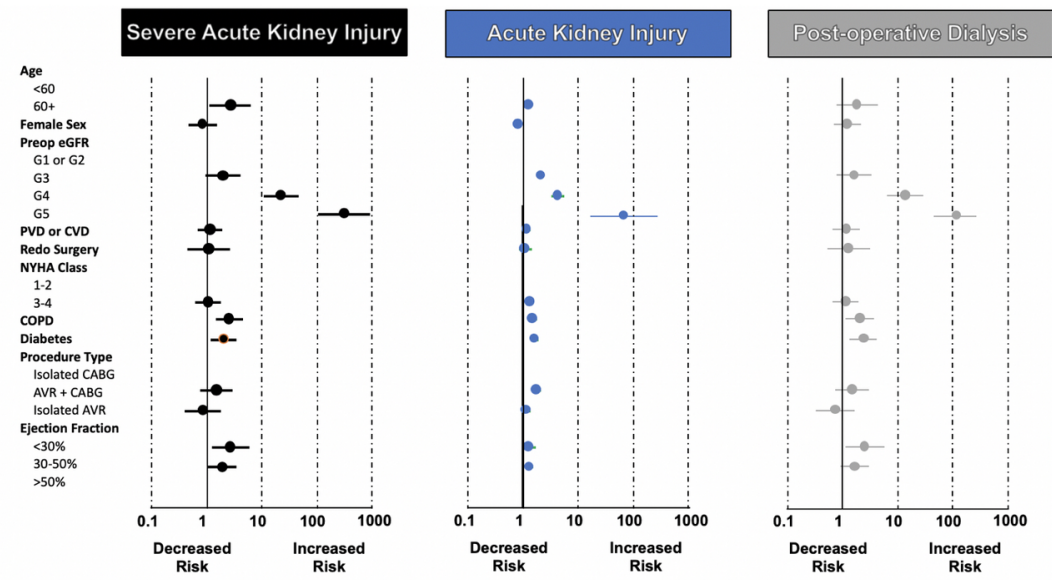

Figure 1. Adjusted predictors of AKI, severe AKI and need for post-operative dialysis after multivariable adjustment (visualized as

relative odds with $95 \%$ confidence intervals). 